\title{
2006-1469: GRADING LAB REPORTS EFFECTIVELY: USING RUBRICS DEVELOPED COLLABORATIVELY BY ECE AND TECHNICAL WRITING INSTRUCTORS
}

\section{Alexis Powe, Mississippi State University}

Alexis Powe is an instructor in the Mississippi State University Bagley College of Engineering's Shackouls Technical Communication Program, where she teaches Technical Writing. She received her BA in English from MSU in 2002 and her MA in English from Louisiana State University in 2004. She previously served as a composition lecturer for the MSU Department of English. Her professional memberships include ASEE and MLA (Modern Language Association). Address: Box 9544, Mississippi State University, Mississippi State, MS, 39762; telephone: (662) 325-4240; fax: (662) 325-7183; e-mail: apowe@engr.msstate.edu.

\section{Jane Moorhead, Mississippi State University}

Jane Moorhead is an instructor at Mississippi State University in the Electrical and Computer Engineering department. She has taught Digital Devices and Logic Design, Microprocessors and Introduction to ECE. She received her BS in EE from North Carolina State University and her MS in Computer Engineering from MSU. She previously worked for IBM as a design engineer in communications products as well as in speech recognition research. Her professional memberships include ASEE and IEEE. Address: Box 9571, Mississippi State University, Mississippi State, MS, 39762; telephone: (662) 325-2096; fax: (662) 325-2298; e-mail: janem@ece.msstate.edu. 


\title{
Grading Lab Reports Effectively: Using Rubrics Developed Collaboratively by ECE and Technical Writing Instructors
}

\begin{abstract}
This paper describes a collaboration between a sophomore/junior-level lab component in an electrical and computer engineering (ECE) course and a junior-level technical writing course within the Mississippi State University Bagley College of Engineering's Shackouls Technical Communication Program (TCP). Grading for labs with weekly writing assignments poses challenges previously overlooked by collaborations between ECE and the TCP. Since lengthy reports are required weekly for the Digital Devices and Logic Design (ECE 3714) lab component, grade turnaround must be quick and can become extremely burdensome for teaching assistants, many of whom struggle with writing themselves due to lack of confidence or ESL challenges. This collaboration differs from other ECE-TCP collaborations significantly in that it utilizes a lab-grading system that combines traditional, non-quantitative rubrics and set penalties for mechanical/stylistic errors to expedite the grading process while retaining grading reliability among TAs. Five specific areas have been defined within each rubric to make up the total percentage of the grade, with the point values changing to reflect the focus of each lab. Topics discussed include an overview of analytic versus holistic grading and the rationale behind the authors' grading approach, previous ECE-TCP collaborations, the combined rubric-set penalties grading system for ECE 3714 with sample grading materials provided, quantitative and qualitative assessments of the newly implemented grading approach, and potential pitfalls of the authors' grading approach.
\end{abstract}

Keywords: analytic grading, collaboration, ECE, holistic grading, inter-rater reliability, technical writing

\section{Introduction}

Engineering educators are under increasing pressure from administrations, Writing across the Curriculum (WAC) programs, writing educators, and industry to incorporate more writing into their classrooms. They rarely debate the need to improve engineering students' written (and oral) communication skills, the benefits of which are well documented. Perhaps the most important skill writing fosters in students is critical thinking. As Wheeler and McDonald state, "Writing, like teaching, forces one to think—hard!"1 However, new educators-both those taking over classes already firmly established by more senior faculty and those creating new classes-struggle to incorporate writing into their classrooms and, even more challenging, to grade that writing effectively. Pappas and Hendricks state, "The problem is not that engineering faculty members fail to value good writing and speaking; the problem is that they are not trained to teach or evaluate these subjects." ${ }^{2}$ Developing an effective—and efficient—grading approach is particularly difficult for new educators who lack confidence in their evaluation abilities or those of their teaching assistants (to whom the grading often falls). Meanwhile, technical writing educators constantly seek to reinforce the importance of writing to students outside of the writing class. Both interests, and ultimately those of students, are served when educators from various engineering departments work with technical writing educators to develop a grading system that provides specific grading criteria for the grader(s) to ensure grading consistency, 
generates constructive feedback for students, allows for quick turnaround by the grader(s), and values both technical content and mechanical/stylistic correctness.

This paper describes a collaboration between a sophomore/junior-level lab component in an electrical and computer engineering (ECE) course and a junior-level technical writing course within the Mississippi State University Bagley College of Engineering's Shackouls Technical Communication Program (TCP). The instructor for Digital Devices and Logic Design (ECE 3714) sought help from the TCP to develop a grading system for a weekly lab component that required students to submit lengthy reports. The ECE instructor identified four major priorities. The first two-accommodating discrete lab elements and increasing inter-rater reliabilitysuggested the need for an analytic grading approach, with set point deductions for each lab component and mechanical/stylistic errors. The other two-fairly evaluating content and reducing grading turnaround time-suggested a holistic grading approach, with rubrics created for each assignment against which reports could be compared to determine the overall grade.

In response, the authors have formed a hybrid lab-grading system that combines traditional, nonquantitative rubrics with quantitative set penalties for discrete lab components and mechanical/stylistic errors to form the overall report grade. This system expedites the grading process while providing structure for the course's three TA graders to maintain inter-rater reliability. Five specific areas have been defined within each rubric to make up the total percentage of the grade, with the point values changing to reflect the focus of each lab. Preliminary results and feedback have been generally positive, with TAs reporting that the rubrics facilitate the grading process, provide a measure of fairness, and allow for fast grade turnaround. The main problem the authors have observed with the newly implemented grading system is that most students do not regularly consult the rubrics (which are posted to the course web site) while writing their lab reports, as the authors intended. The authors surmise that this behavior results from procrastination, apathy, or ignorance (some students claimed that they were unaware that the rubrics existed or that they were posted to the web site, despite repeated class announcements and an optional information session conducted to address students' questions about the rubrics). The authors encourage new engineering educators teaching similar courses to adapt this grading system for their own use.

Topics discussed include an overview of analytic versus holistic grading and the rationale behind the authors' grading approach, previous ECE-TCP collaborations, the combined rubric-set penalties grading system for ECE 3714 with sample grading materials provided, preliminary quantitative and qualitative assessments of the study, and potential pitfalls of the authors' grading approach.

\section{Background and rationale}

To understand this hybrid grading approach, new engineering educators need a working knowledge of analytic versus holistic grading approaches.

Simply speaking, an analytic grader deducts points from the writer's overall grade based on predetermined values assigned to each aspect of the written document, from content to mechanics. For example, many composition programs (from elementary schools to universities) 
have provided or continue to provide their graders with lists of mechanical/stylistic errors and corresponding penalties for each error. The major benefit to this grading approach is inter-rater reliability (which is especially important among inexperienced graders, like teaching assistants), lending a semblance of objectivity to an inherently subjective grading process and reducing the learning curve for new graders.

Holistic grading offers an entirely different evaluative tactic. Pappas and Hendricks clearly define the holistic approach:

Holistic grading examines a document or presentation as a whole rather than considering technical content and writing style (including grammar and mechanics) as separate entities. In particular, holistic grading does not follow the usual formula for deducting points for various grammatical or stylistic errors. Rather, it defines standards for various grades against which the entire document or presentation is judged. In particular, an effective written document or oral presentation must be evaluated in the context of the purpose for which it is intended, and the degree of success with which it satisfies this purpose $[\ldots .$.$] Engineering faculty may be more comfortable with and adept at grading$ along such workplace standards. ${ }^{2}$

Holistic grading uses a rubric to establish grading criteria for each assignment, against which the grader(s) compares the assignments and judges them accordingly. Sample rubrics and a discussion of holistic grading by White are useful in understanding the holistic grading movement. ${ }^{3}$

As is true of any grading approach, holistic grading poses some difficulties. DuVal writes, "Most English teachers employ holism every time they read a student paper. Experience teaches them exactly what grade a high school, GED, or college essay merits without making a single mark on the paper. It is for the student's sake that the red ink is spilled."4 The difficulty, however, is that new engineering educators are not trained English teachers and are often inexperienced graders of writing. This is doubly true of their TAs, who frequently grade undergraduate documents. Also problematic is the inter-rater reliability of holistic evaluation. Bacha, who compared analytic and holistic assessment methods in an English as a second language (ESL) program, acknowledges that analytic assessment methods offer high inter-rater reliability and that holistic grading can be construed as "focus[ing more] on what the writer does well rather than on the writer's specific areas of weakness." ${ }^{5}$ Bacha concludes:

Although holistic scoring may blend together many of the traits assessed separately in analytic scoring, making it relatively easier and reliable, it is not as informative for the learning situation as analytic scoring [....] More attention should be given to the language and vocabulary aspects of students' essay writing and a combination of holistic and analytic evaluation is needed to better evaluate students' essay writing proficiency at the end of a course of study. ${ }^{5}$

While holistic grading — when done conscientiously_ can be a valuable and efficient approach, strictly holistic grading is inappropriate for every assignment. Pappas and Hendricks, who propose a strictly holistic grading method for "day-to-day communications" that "require no more than an unambiguous understanding of what the author is communicating," argue certain types of documents, presumably including formal reports, call for "'academic' English usage and style", evaluation. ${ }^{2}$ 
Based on these studies and previous collaborations between ECE and the TCP at Mississippi State University, the authors determined that a hybrid grading approach with both analytic and holistic qualities best suited ECE 3714.

\section{Previous ECE-TCP collaborations}

This effort draws from previous collaborations between the Bagley College's ECE department and the TCP in three courses: Senior Design I (ECE 4512/4532), Intermediate Electronic Circuits (ECE 3424), and Advanced Electronic Circuits (ECE 3434). The first collaboration combined a section of Technical Writing (GE 3513) with ECE Senior Design I because both courses are writing intensive. ${ }^{6}$ While this article draws nothing specific from this collaboration, the effort fostered future collaborations between the TCP and ECE programs that produced nonquantitative rubrics for ECE assignments. For ECE 3424 and 3434, ECE and TCP educators jointly created templates and non-quantitative rubrics for written assignments (each class required an interim and a final report) similar to those used in Technical Writing. ECE and TCP educators equally initiated these collaborations to provide grading standards and reinforce the importance of WAC. In all three courses, ECE professors specified the content requirements, while TCP instructors helped develop a template and rubric for each assignment based on those specifications. The templates helped students understand the content requirements of each assignment, and the rubrics eased the grading process for ECE TAs. Figure 1 shows the rubric used in ECE 3434 (inspired by Pappas and Hendricks ${ }^{2}$ ).

ECE 3434 Lab Report Rubric (Interim and Final Reports)

D $(\mathrm{D}+/ 68, \mathrm{D} / 65, \mathrm{D}-/ 62$; all points are out of 100):

- Meets the basic guidelines for the assignment in question regarding fonts, margins, title page, table of contents, introduction, body, conclusion and references if used.

- Attempts to include all specified content areas in the body.

- For the interim report, this includes the following: (1) schematic diagram, (2) description of progress in building the prototype, (3) discussion of problems and remedies, (4) description of the basic operation and function of the various subcircuits of the given amplifier design, and (5) comparison of the Lin architecture of the audio amplifier with that of a 741 operational amplifier and the LM380 IC power amplifier.

- For the final report, this includes the five criteria above as well as the following: (1) incorporation of the expanded work from labs 9-11 and the lab notebook, (2) description of the testing and performance of the finished prototype, and (3) improvement upon the interim report in content and style.

- Contains no grievous errors regarding use of source material (does not create the impression of plagiarism).

- Does not destroy readers' confidence with numerous grammatical/mechanical errors.

C $(\mathrm{C}+/ 78, \mathrm{C} / 75, \mathrm{C}-/ 72)$ - Meets D criteria and also does the following:

- $\quad$ Targets the appropriate audience (engineering managers and customers).

- Employs an effective system of headings in the body of the report.

- Attempts a suitable report introduction-subject, purpose, scope, preview of organization, list of references (if applicable), and various optional elements.

- Attempts a suitable conclusion—reiterates key points and attempts to provide insight into these points. 
- Has few grammatical/mechanical errors (especially serious ones—see A).

B $(\mathrm{B}+/ 88, \mathrm{~B} / 85, \mathrm{~B}-/ 82)-$ Meets $\mathrm{C}$ criteria and also does the following:

- Attempts to cultivate a professional writing style: few choppy sentences, appropriate transitions between ideas/sentences/paragraphs, appropriately formal tone, nice organization and flow of ideas.

- Provides plenty of detail in explaining methods, setbacks, etc.

- Avoids second person (you, your, yourself, etc.) altogether and limits first person (I, my, our) whenever possible.

- Is mostly successful with use/integration of graphics (numbered, titled, cited if necessary, mentioned in the text, and positioned near first textual reference).

- Has few grammatical/mechanical errors (especially serious ones-see A).

A $(\mathrm{A}+/ 98, \mathrm{~A} / 95, \mathrm{~A}-192)$ - Meets $\mathrm{B}$ criteria and also does the following:

- Is appropriately and consistently organized; body sections are discrete.

- Incorporates methods nicely (provides plenty of detail, numbers steps, uses indicative mood, and uses active or passive voice consistently).

- Manages IEEE reference style with no trouble.

- Has very few, if any, grammatical/mechanical errors, especially serious ones (verb errors, sentence fragments, comma splices, misspellings, incomprehensibly mixed constructions, etc.).

(NOTE: Pluses and minuses depend on how fully the paper meets the criteria for a particular category.)

Figure 1. Rubric developed for interim and final reports in ECE 3434

Implementing a similarly holistic grading approach in Digital Devices and Logic Design (ECE 3714), however, was difficult for four reasons:

- Rubrics in previous collaborations were designed to evaluate the written document as a whole, not documents containing discrete elements independently responsible for a portion of the students' overall grades. In ECE 3714, each lab report contained discrete elements, such as pre-lab and in-class data sheets, and content requirements were weighted differently from assignment to assignment.

- Inter-rater reliability was a greater concern in ECE 3714. Whereas ECE 3424 and ECE 3434 each utilized one TA as the sole grader, ECE 3714 reports were divided among three TAs, all of whom lacked previous grading experience and struggled with writing themselves due to lack of confidence. Also, ECE 3714 TAs expressed concerns about marking and comparing mechanical errors to a rubric that specified, for example, that an "A"-range paper have few serious mechanical errors. The TAs often struggled to determine the severity of mechanical errors and penalized subjectively, resulting in student complaints. For example, some TAs considered misspelled words negligible errors and deducted few, if any, points, while others considered them major errors and penalized heavily.

- $\quad$ ECE 3714 students needed a clearer understanding of how their grades were calculated and the instructor's expectations of content and mechanics, as well as the deductions taken for errors in the latter category. This understanding was also needed to ensure grading reliability and to address instances where TAs might mistakenly deduct points for an error where none existed. 
- $\quad$ Faster grade turnaround was necessary in ECE 3714 because lengthy reports (12 total) were collected weekly, whereas ECE 3424 and ECE 3434 required only two reports (interim and final).

The strictly holistic grading approach was clearly ill fitted to ECE 3714 and needed customization.

\section{Description of newly implemented grading approach}

In response to the four abovementioned difficulties, ECE and TCP instructors developed a basic rubric that divides points among five separate categories, facilitating the grading of discrete elements in labs. Within the four content-based categories, the ECE instructor provides traditionally non-quantitative holistic guidelines specific to each part of each assignment. The fifth category specifically addresses mechanical/stylistic correctness, which counts 15 percent of the total grade for the first two labs and 10 percent for the remaining labs. (These percentages were co-determined by the ECE and TCP instructors to be lenient enough to prevent mechanical/stylistic correctness from becoming an overriding concern, yet punitive enough to reinforce the importance of mechanical/stylistic correctness, especially early in the semester.) The fifth category utilizes specific, quantitative penalties for mechanical/stylistic errors to ensure inter-rater reliability. Figure 2 shows a sample rubric, and Figure 3 shows the set penalties for specific mechanical/stylistic errors.

\footnotetext{
Lab Report 7-Social Security Number Decoder (Combinatorial System Design)

I. Pre-lab Data Sheet (20 percent of overall grade)

A/Full credit (20 points):

The student has correctly completed all components of the pre-lab data sheet before the lab and has a hard copy with him/her (or an electronic version immediately accessible on his/her laptop).

For Part 1:

- Has a truth table, a Karnaugh map (Kmap), and a minimum SOP equation for all four functions

- Has .gdf schematic circuits for all four functions

- Has simulations for all four functions

- Has protoboard layout of circuits clearly indicating pin assignments, including switch and LED connections
}

For Part 2:

- Has VHDL code written and simulated in MAXPLUS II

- Shows that all four simulations match the expected outputs in the truth tables

C/Half credit (10 points):

The student has correctly completed some, but not all, of the pre-lab data sheet components before the lab (and/or has shown a genuine attempt to do so correctly) and has a hard copy with him/her (or an electronic version immediately accessible on his/her laptop). The student has completed approximately half of the abovementioned requirements.

F/No credit (0 points):

The student has completed very few or none of the abovementioned data sheet requirements and/or produces no paper or electronic version at the beginning of the lab. 
II. In-lab Data Sheet (30 percent of overall grade)

A/Full credit (30 points):

The student has correctly completed all components of the in-lab data sheet before the end of the lab and shows his/her work to the TA before leaving.

For Part 1

- Has all four circuits wired and working simultaneously

- Uses short wires to minimize "rats' nests"

- Has wired circuits with little or no TA assistance

- Has debugged errors with little or no TA assistance

- Is able to communicate procedures and encountered problems to the TA or instructor clearly and knowledgeably

For Part 2:

- Has PLD programmed on Warp to implement all four functions

- Has PLD wired with switches and LEDs

- Shows TA that all four functions work properly

C/Half credit (15 points):

The student has correctly completed some, but not all, of the in-lab components before the lab ends (and/or has shown a genuine attempt to do so correctly) and shows his/her work to the TA. (The student has completed approximately half of the abovementioned requirements.)

F/No credit (0 points):

The student has completed very few or none of the abovementioned requirements and/or does not show work to the TA.

\section{Report Content (30 percent of overall grade)}

A/Full credit (30 points):

- $\quad$ The report contains a descriptive abstract (that includes the equipment used in the lab) and is 3-4 lines long.

- The report shows that the student has a thorough understanding of the lab's purpose and the concepts discussed in relation to the lab.

- $\quad$ The report shows that the student can clearly communicate the lab techniques utilized.

- $\quad$ The report diagrams are in MAXPLUS II (or something similar) rather than hand-drawn.

- $\quad$ The report includes a table of data verifying that the expected results were obtained.

- The report includes a discussion of Kmaps, simplification of functions using Kmaps, VHDL, and usage of VHDL versus schematic capture software.

- The report properly incorporates outside sources as well as more in-depth information in the theory portion of the lab.

- The report targets an appropriately technical audience and is formal in tone.

C/Half credit (15 points):

- The report contains an abstract, although it may not be entirely appropriate to the assignment or mention equipment used in the lab.

- The report shows some understanding of the lab's purpose and the concepts discussed in relation to the lab.

- The report reveals that the student has difficulty clearly conveying the lab techniques utilized (shows some lack of understanding).

- The report diagrams are hand-drawn, but accurate.

- The report includes a table clearly explaining the lab results and attempts discussion of the elements listed in $\mathbf{A}$.

- The report incorporates few additional references or does so problematically and does not examine the theory in depth. 


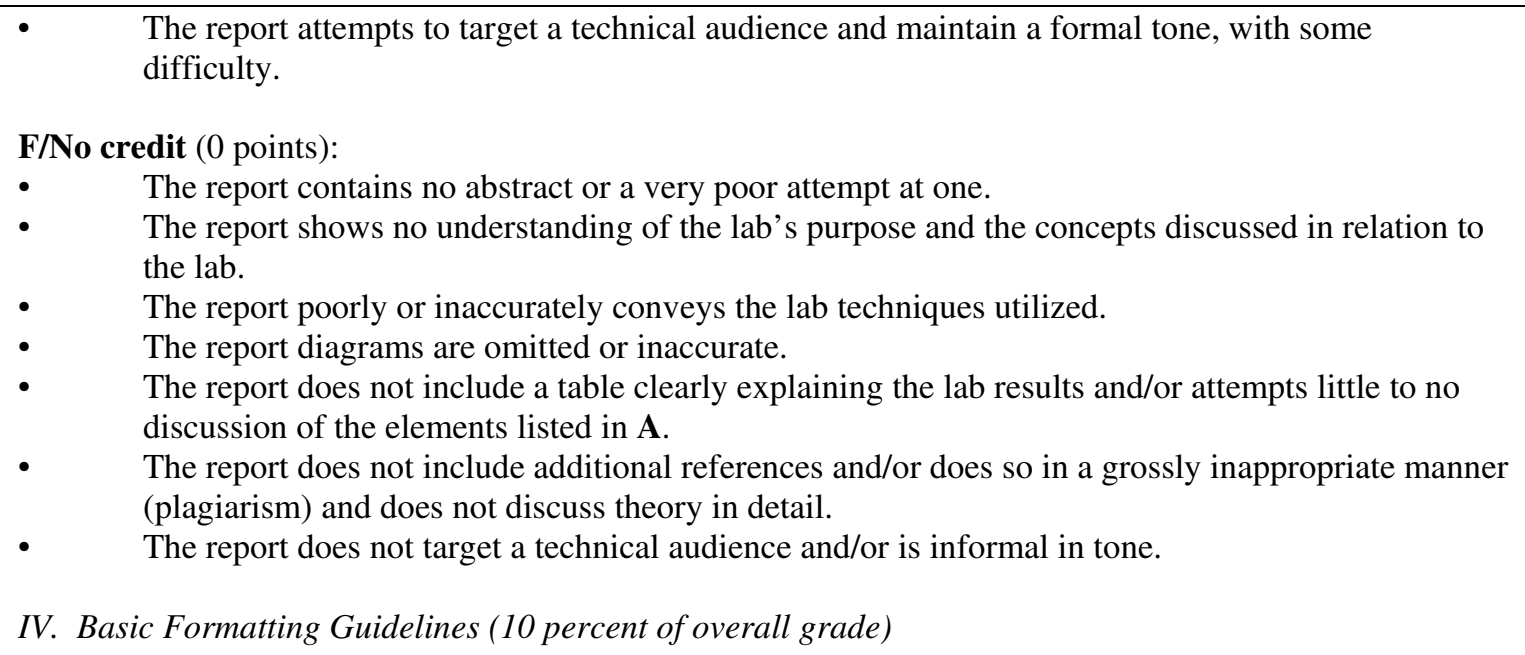

IV. Basic Formatting Guidelines (10 percent of overall grade)

A/Full credit (10 points):

The report follows all guidelines regarding fonts, margins, title and headers. The report is submitted in PDF format and on time.

C/Half credit (5 points):

The report meets some of the formatting guidelines, but not all.

F/No credit ( 0 points):

The report shows no effort to follow formatting guidelines.

V. Mechanical/Stylistic Correctness (10 percent of overall grade)

Refer to the mechanics sheet for quantitative deductions (up to 10 points deducted for mechanics and style).

Figure 2. Lab report 7 rubric for ECE 3714

\section{Mechanical/Stylistic Errors Grade Sheet}

Lab:

Grader:

Student's Name:

Assignment \#:

Category 3: Severe Errors (3 points each)

Mixed sentence construction (i.e., incomprehensible sentence)

Subject-verb disagreement

Comma splice

Fused sentence ("run-on")

Sentence fragment

End-sentence punctuation omission

Double negative

Verb error

Misspelled word

Word omission (severe) 


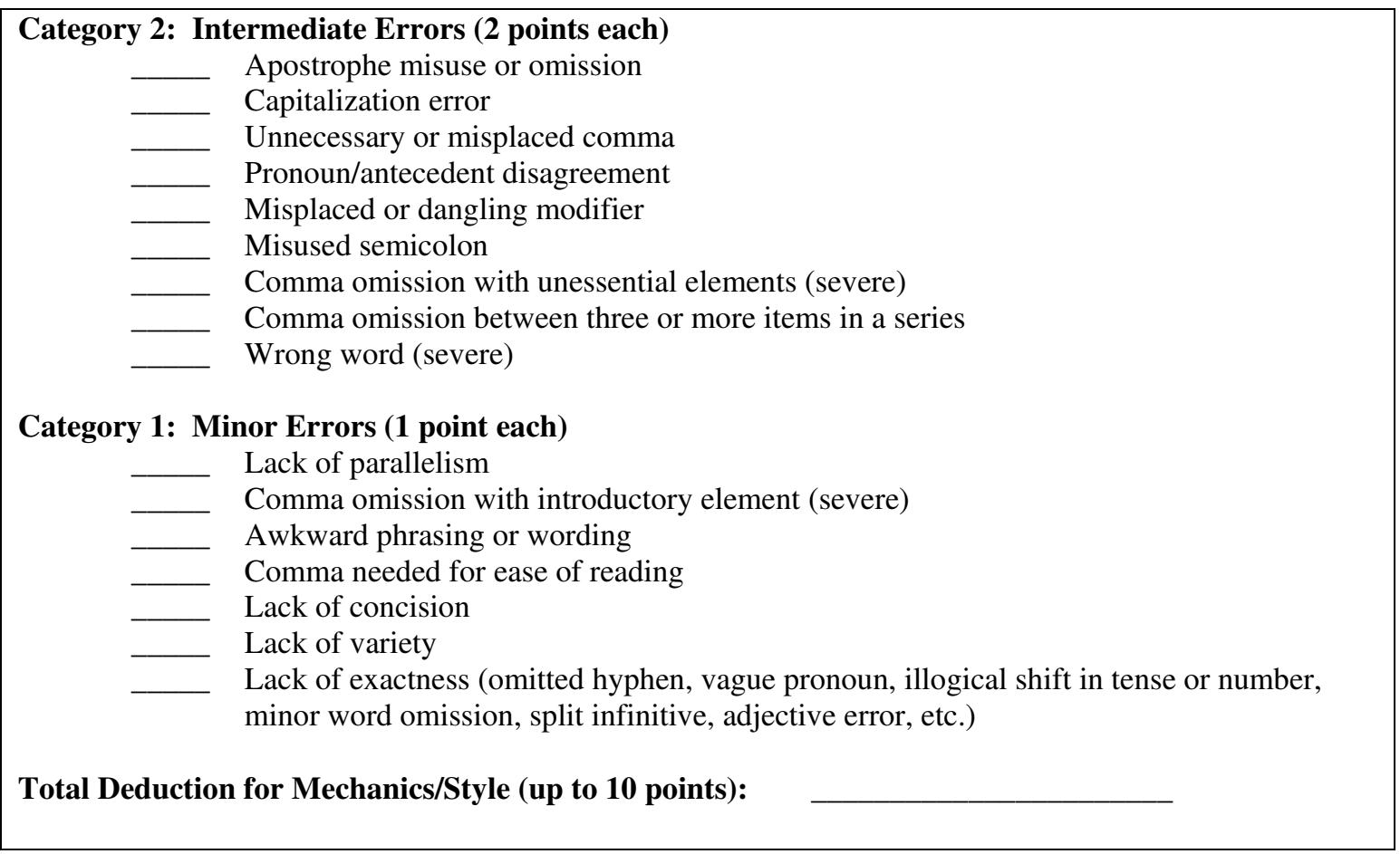

Figure 3. Set penalties for mechanical/stylistic errors (closely adapted from [8])

The general rubric is tailored to each assignment to reflect changing point values from assignment to assignment. By providing the ECE 3714 students with the rubric for each assignment, the ECE instructor serves a number of needs. Students gain a better understanding of the instructor's and TAs' expectations regarding the content of each lab and of their grades on each lab. If there is a dispute among a student and a TA (over a mechanical error, for example), the student can report the grading error to the TA and the deducted points can be restored easily, which is difficult to do in a rubric-based grading system. This last point is also extremely important in programs, like that at MSU, where TAs struggle with writing and mechanics themselves, due mainly to lack of confidence among native English speakers and to ESL challenges among non-native English speakers. This way if a grader mistakenly deducts three points for a mechanical error where none exists, for example, the student can easily spot the mistake and have it corrected to his or her satisfaction. Finally, the primarily holistic thrust of the rubrics expedites lab-report grading.

\section{Assessment}

This section provides quantitative and qualitative assessments of the new grading approach solicited by the authors, who acknowledge that results are preliminary and warrant continued research. The quantitative assessments draw from surveys (attached as appendices A and B) distributed to ECE 3714 students and TAs, though the student survey results were problematic and discarded. The authors also provide average grades for reports 1-4 to help gauge inter-rater reliability.

The student survey results are invalid as the survey revealed that a vast majority of students had rarely or never reviewed the grading rubrics. After admitting this in the first question, students 
answered the remaining survey questions, thus invalidating the results. The authors estimate (based on their reviews of the survey responses, qualitative written comments by students, and informal class polls) that only one-fourth of students surveyed had reviewed the rubrics regularly. However, since the surveys were anonymous and the authors were unable to verify this estimate, they discarded the survey results. Student comments in relation to the survey reveal three causes for their disinterest in the rubrics: procrastination, apathy, and ignorance. Regarding the last cause, some students claimed ignorance of the rubrics even though the instructor had posted them to the course web site and announced an optional information session to address students' questions about the lab grading approach.

TA feedback to the rubrics has been overwhelmingly positive. The average responses by the three TAs to the five survey questions (using traditional Likert scales) are 4.67, 4.67, 4.00, 3.33, and 1.67 .

Figure 4 reveals expected grade variations among sections, but overall no TA is grading exceedingly harshly or leniently, providing reasonable inter-rater reliability. Average grades for reports $1-4$ in each section are $86.14,90.02,85.69,84.34$, respectively, revealing less than a sixpoint variation among graders.

\begin{tabular}{|l|l|l|l|l|}
\hline $\begin{array}{l}\text { Grader } \\
\text { for } \\
\text { section }\end{array}$ & Lab 1 & Lab 2 & Lab 3 & Lab 4 \\
\hline TA 1 & 89.39 & 74.39 & 86.84 & 93.94 \\
\hline TA 1 & 92.06 & 78.81 & 95.54 & 93.68 \\
\hline TA 2 & 82.50 & 94.38 & 76.86 & 89.00 \\
\hline TA 3 & 89.89 & 94.89 & 79.78 & 72.78 \\
\hline
\end{tabular}

Figure 4. Average grades on lab reports 1-4

As qualitative measures of the study's success, the authors solicited additional written feedback on the surveys of students and TAs. The following section categorizes those comments based on the four objectives the authors sought to alleviate with the hybrid grading approach: the ability to grade labs that contain discrete elements (such as pre-lab and in-lab data sheets), improved inter-rater reliability among the three ECE 3714 TA graders, better student understanding of the grading standards utilized in the course, and faster grade turnaround. (Comments are taken verbatim from the surveys.)

The grading approach accomplishes the first goal fairly easily by creating mini-rubrics within the larger rubric for discrete lab elements, though TA 1 made the following comment about sections III, IV, and V of the rubric: "As it stands, the rubrics need to be updated to focus only on whole portions of the lab, e.g., the ENTIRE lab report is worth $30 \%$. Splitting up the grading to focus on PARTS of the lab report, e.g., grammar is $10 \%$, formatting is $10 \%$, etc., is too detailed for the objectives of this course."

Regarding improved inter-rater reliability, one student commented that his/her TA "was very easy on grading." TA 1 commented that "It [the rubric for an assignment] helps to see the main points of what we're looking for when grading." TA 2 commented, "It's very easy to use the 
rubrics to see how much each section of the lab should count. I don't have to spend time trying to decide which parts of lab are more important-I just look at the rubric and can assign points." TA 2 added later, "By using the rubrics everyone is graded on the same scale, no matter who their TA is. This makes the system very fair, in my opinion." TA 3 commented that "They [rubrics] help me," but for the first time expressed to the authors his reluctance to abide by the rubrics:

There's things I supplement "on the fly" while I grade lab reports. Like how much [sic] certain misconceptions the student has about particular concepts. I do a lot of broad generalization of what I think the lab report grade should be. This might be because the rubrics tend not to designate points for specific information the lab report has in it [...] I don't usually follow the guideline for assigning a grade. Sometimes I feel the rubric does not take off enough points for things that should be in the lab, sometimes I feel it is too harsh on some things.

TA 3's reluctance has necessitated further discussions between the authors and the TAs about the importance of TA participation in the rubric-building process and the necessity of grading based on the final rubric produced by that collaboration.

Regarding students' understanding of the grading system, one student commented, "they [rubrics] are helpful, but prelabs seems [sic] misunderstanding." Another student wrote that "more detail on the early rubrics would be more helpful." Two TAs expressed similar sentiments that they "can't tell one way or another if the students are looking at rubrics." TA 1 elaborated, "What I do see is that the overall trend of poor students doing poorly and good students doing well remains, no matter what portion of the lab is being referred to. I think this trend will continue no matter what policies are in place." TA 2 commented that "if I tell them [students] 'this prelab is worth 50 points' they generally have it done, or have made a good attempt and [sic] finishing it." TA 3 commented that "if they [students] really used the rubric everybody would get a 100. This of course is not the case. I do think it [the rubric] helps the students focus their efforts in preparing a lab report and what portions of the lab are important to do." None of the TAs reported grade complaints from students.

Finally, the fourth objective - faster grade turnaround - has been met. For the first time in the ECE 3714 lab sections overseen by the author, two of the three TAs have consistently returned lab reports within one week's time, with the third TA lagging a few days behind.

\section{Discussion of potential pitfalls}

This section targets new engineering educators who are considering using the proposed grading approach in their classes. As with any approach to grading writing, this one is not without difficulties. Within each area listed below, the authors identify potential pitfalls to their grading approach that they have encountered and ways that new educators may mitigate these concerns.

\section{A. Developing rubrics}

Graders often evaluate papers based on implicit standards, but the value of making those standards explicit in a rubric is immense, standardizing grading between individuals and controlling each grader's fluctuations based on mood or sleep deprivation. Many rubricdevelopment tools exist to aid first-time rubric creators, including those referenced in this article 
and online resources. ${ }^{9}$ However, the authors find that the criteria to include on a rubric and the distinctions between letter grades are largely based on the writing assignment specifications, the complexity of the material tackled, and trial and error. One also may utilize templates and student-written examples of good and bad papers. The authors also encourage educators to involve students in the rubric-development process.

\section{B. Distributing rubrics to students}

Educators who grade using rubrics face a difficult decision: whether or not to distribute the rubrics to students before the assignment is submitted for grading. Some educators develop rubrics for private use and do not distribute them to students, opting to discuss the rubrics with students only upon request or not at all, whereas others believe that the purpose of creating rubrics is to share them with students.

One reason educators may decide against distributing rubrics to students is the specificity of the rubrics. Highly specific rubrics contain exact information, sometimes in minute detail, that should appear in an "A" paper, "B" paper, etc. One may argue that distributing such specific rubrics to students negates the challenge of writing. Good writers must judge for themselves the importance/relevance of information and the best way to organize the information, basing these decisions on the writing assignment, on the educator's instructions regarding content/format, and on basic trial and error. In other words, distribution of highly detailed rubrics to students contradicts the purpose of the writing assignment: to develop critical thinking skills in students. Another disadvantage of distributing rubrics to students is that students occasionally interpret the rubrics differently from the grader and, if dissatisfied with their grades, challenge them. However, Bloede ${ }^{10}$, Boyd and Hassett ${ }^{11}$, and Pappas and Hendricks ${ }^{2}$ all report success with distributing rubrics to students and claim that doing so actually reduces student grade complaints.

Other educators not only distribute rubrics to students before collecting assignments, but also involve students in creating them. The authors of this article chose to share rubrics with students during the second semester of the study to solicit student interaction and feedback. TAs report no grade complaints.

For those educators debating sharing rubrics or keeping them private, a third solution presents itself: involving students in creating a generalized rubric at the beginning of the semester. This option provides students an intimate understanding of the grading process without thwarting the development of critical thinking skills. Also, after developing a basic, comprehensive rubric, the instructor may solicit student input in tailoring the rubric to later assignments through class discussion.

\section{Improving TA grading and inter-rater reliability}

First, one must note that grading writing - whether analytically or holistically — is always at least partially subjective. A strictly objective grading approach for writing is infeasible, which should be understood by all parties (educators, graders, and students) from the class onset. However, faculty and TAs should strive for as much inter-rater reliability as possible. Faculty members bear the responsibilities of providing and thoroughly explaining clear objectives for each written assignment, which are accomplished through comprehensive templates and rubrics, and of 
assisting TAs with grading, thereby easing the grading process for TAs and minimizing the learning curve.

Before the grading begins, faculty members must take steps to prepare TAs for the rigors of grading. Too often TAs are left to their own devices. Perhaps nothing is more bewildering to a new TA than being handed a stack of papers and a rubric and asked to return papers within a week with grades assigned. TAs need to work closely with either an ECE or TCP instructor to observe an experienced grader firsthand. Inter-rater reliability may be improved by TAs holding grading sessions after collecting each assignment, during which they exchange two or three sample papers and grade them independently of one another before comparing the results and discussing any discrepancies. This practice is especially important early in the semester. While grades will certainly vary at least slightly, the goal is to ensure that each TA is aware of the approximate levels at which the others are grading and adjusts his/her practices accordingly. If two TAs assign a paper wildly varied grades, then the faculty instructor may adjudicate the grade. These sessions can be held in a mandatory class that meets once or twice weekly and that possibly begins a few weeks before labs do to allow students to practice on sample papers from previous semesters. Beginning the meetings before the regular semester also provides the instructor time to initiate a basic grammar review and to teach TAs the proper names for mechanical/stylistic errors so that they can later properly categorize errors and deduct points based on the errors' severity.

Also important is ongoing instruction in mechanical/stylistic correctness for TAs. Learning proper names for mechanical errors is one of the most challenging and most important tasks for new TAs evaluating writing, and a TCP or English department instructor can easily complete a few training sessions on the subject if the ECE instructor feels uncomfortable or ill-equipped to do so. Brocato et al. recommend that potential TAs take a grammar test before grading begins to ensure they are capable of recognizing basic writing errors and, if they perform poorly, complete a grammar review course before retaking the test. Brocato et al. provided TAs a sample lab report as a screening exam and asked them to identify both "high-order writing concerns related to writing style, including overly repetitious words/phrases, general wordiness, lack of parallelism, vague subjects, unnecessarily inactive verbs, and inconsistency in writing numbers" and "low-order writing concerns, many of which were grammatical in nature, and included misspelled words, misuse of first person, faulty paragraph division, use of slang, and misuse/omission of articles." 12 Brocato et al. also enrolled TAs with ESL concerns in a specialized ESL course devoted to improving TA grading. When retested later in the semester, TAs who had enrolled in the ESL class showed a 57 percent average improvement on the grammar test. ${ }^{12}$ For continued support throughout the semester, TAs may work with technical writing tutors and possibly graduate students enrolled in English or technical writing programs. This collaboration benefits all involved, providing valuable experience to English students considering pursuing teaching or technical writing careers and possibly financial assistance for English graduate students, who are chronically underfunded.

Finally, quantitative penalties for mechanical/stylistic errors can prove problematic. For example, if a TA spots a misspelled word that reappears multiple times in a report, he or she may choose to count the word as misspelled only once. Another TA in the same situation may choose to deduct points for each offense. These issues may be discussed and settled by TAs and the 
overseeing instructor before grading begins and during weekly grading sessions to ensure interrater reliability.

\section{Providing written feedback on student papers}

To reduce turnaround time, holistic graders sometimes decide against providing extensive written comments on papers. The authors strongly discourage this practice as detrimental to the students' development as writers, as do most writing evaluators. ${ }^{2,4}$

\section{Conclusion}

As the preliminary assessment shows, the proposed grading system allows for a partially holistic grading approach to formal writing that contains discrete elements, improved inter-rater reliability, a better understanding of the grading system in ECE 3714 for TAs (feedback on improved student understanding was inconclusive), and faster grade turnaround. The authors hope to improve this grading approach in future semesters and potentially help other engineering educators adapt it to their classes' lab components.

\section{Acknowledgements}

The authors thank John Brocato, Bill Chapman, Marion Hagler, Jim Harden, Joe Picone, and Bob Reese for initiating collaborations between the MSU Bagley College of Engineering's Shackouls Technical Communication Program and the Department of Electrical and Computer Engineering, upon which this collaboration builds. The authors also thank John Brocato for his guidance during the writing process and Kelly Rimel, Courtnay Greer, and Adrienne Howse for their assistance with final draft preparation.

\section{References}

[1] E. Wheeler and R.L. McDonald, "Writing in engineering courses," Journal of Engineering Education, vol. 89, pp. 481-486, Oct. 2000.

[2] E.C. Pappas and R.W. Hendricks, "Holistic grading in science and engineering," Journal of Engineering Education, vol. 89, pp. 403-408, Oct. 2000.

[3] E.M. White, Assigning, Responding, Evaluating: A Writing Teacher's Guide, $3^{\text {rd }}$ ed., Boston, MA: Bedford/St. Martin's, 1999.

[4] K.N. DuVal, "Holistic grading and the marking myth: What's so bad about a little red ink?" Adult Learning, vol. 7, pp. 11-12, May/June 1996.

[5] N. Bacha, "Writing evaluation: What can analytic versus holistic essay scoring tell us?" System: An International Journal of Educational Technology and Applied Linguistics, vol. 29, pp. 371-83, Sept. 2001.

[6] J. Brocato and J. Picone, "Writing while designing: Combining ECE senior design with an existing technical writing course," in Proc. ASEE Southeast Section Conference, Chattanooga, 2005.

[7] M. Markel, Technical Communication, $7^{\text {th }}$ ed. Boston, MA: Bedford/St. Martin's, 2004, pp. 495-496.

[8] Department of English, Mississippi State University, "Mechanics grade sheet," unpublished.

[9] C.S. Holzberg and S. Brooks-Young, "Worksheets and templates," Technology \& Learning, vol. 24, p. 38, May 2004. 
[10] R. Bloede, "Rubrics for drafting and engineering classes," Tech Directions, vol. 60, pp. 30-31, Dec. 2000.

[11] G. Boyd and M.F. Hassett, "Developing critical writing skills in engineering and technology students," Journal of Engineering Education, vol. 89, pp. 409-412, Oct. 2000.

[12] J. Brocato, B. Chapman, and J. Harden, "Improving the writing-evaluation abilities of graduate teaching assistants in ECE labs," in Proc. ASEE Annual Conference \& Exposition, Portland, 2005.

\section{Appendix A}

\section{Survey of Digital Devices and Logic Design (ECE 3714) Students Concerning Rubrics}

Of the six labs completed to date, for how many labs have you used the grading rubrics to prepare?

For each of the following statements, rank your responses to each question by circling 1 to 5 .

$1=$ Strongly Disagree

$2=$ Disagree

$3=$ Neither Agree nor Disagree

$4=$ Agree

5=Strongly Agree

1. The lab rubrics clearly indicate the graders' expectations for each lab.

$\begin{array}{lllll}1 & 2 & 3 & 4 & 5\end{array}$

Additional comments:

2. The rubrics themselves are clearly written and sufficiently detailed.

$\begin{array}{lllll}1 & 2 & 3 & 4 & 5\end{array}$

Additional comments:

3. The percentage of points assigned to each lab's subcategories is fair.

$\begin{array}{lllll}1 & 2 & 3 & 4 & 5\end{array}$

Additional comments:

4. The rubrics are helpful when preparing for the labs.

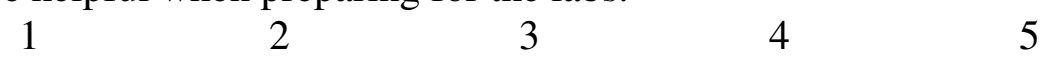

Additional comments:

5. The grades assigned by the TAs are fair when measured against the corresponding rubrics.
1
2
3
4
5

Additional comments:

6. The lab reports are graded consistently between TAs (meaning that all of the TAs are grading at approximately the same levels). 


$\begin{array}{lllll}1 & 2 & 3 & 4 & 5\end{array}$

Additional comments:

Please describe changes that you would like to see to the rubrics or to the grading approach to the labs in this course.

\section{Appendix B}

\section{Survey of Digital Devices and Logic Design (ECE 3714) TAs Concerning Rubrics}

For each of the following statements, rank you response to each question by circling 1 to 5 . $1=$ Strongly Disagree

$2=$ Disagree

$3=$ Neither Agree or Disagree

$4=$ Agree

5=Strongly Agree

1. The rubrics give you a clear understanding of the grading expectations for each lab.

$\begin{array}{lllll}1 & 2 & 3 & 4 & 5\end{array}$

Additional comments:

2. The rubrics help the grading process instead of hinder it.

$\begin{array}{lllll}1 & 2 & 3 & 4 & 5\end{array}$

Additional comments:

3. The rubrics provide a fair grading system for the labs.

$\begin{array}{lllll}1 & 2 & 3 & 4 & 5\end{array}$

Additional comments:

4. The labs clearly indicate that the students are using the rubrics.

$\begin{array}{llllll}1 & 2 & 3 & 4 & 5\end{array}$

Additional comments:

5. The students provide positive feedback about the rubrics.

$\begin{array}{lllll}1 & 2 & 3 & 4 & 5\end{array}$

Additional comments:

Please describe changes that you would like to see to the rubrics or to the grading approach to the labs in this course. 